prevalence, phenomenology and risk factors.

Brain, 123, 733-745. doi:10.1093/brain/

123.4.733.

McKeith, I. G. et al. (2005). Diagnosis and management of dementia with Lewy bodies. Third Report of the DLB Consortium. Neurology, 65, 1863-1872. doi:10.1212/01.wnl.0000187889.17253.b1.

Menon, G. J. (2005). Complex visual hallucinations in the visually impaired: a structured history-taking approach. Archives of Ophthalmology, 123, 349-355. doi:10.1001/archopht.123.3.349.

Mosimann, U. P. et al. (2006). Characteristics of visual hallucinations in Parkinson disease dementia and dementia with lewy bodies. American fournal of Geriatric Psychiatry, 14, 153-160. doi:10.1097/01.JGP.0000192480. 89813.80 .

Teunisse, R. J., Cruysberg, J. R., Hoefnagels, W. H., Verbeek, A. L. and Zitman, F. G. (1996). Visual hallucinations in psychologically normal people: Charles Bonnet's syndrome. Lancet, 347, 794-797. doi:10.1016/S0140-6736(96) 90869-7.

doi:10.1017/S1041610211000809

\section{Musical hallucination in acquired and pre-lingual deafness}

There is little in the literature on auditory hallucinations in the deaf, although the phenomenon is obviously genuine. Bearing this in mind there is hardly anything on non-verbal hallucinations, let alone musical ones.

It is generally accepted that ear disease can give rise to musical hallucination. The most commonly reported ear disease is deafness, which is true especially in the elderly (Pasquini and Cole, 1997). We conducted a Medline search of the relevant literature and found 28 papers (1978-2009) associating musical hallucinations and deafness. Most papers were case reports of one or more cases of psychiatric, neurological and ear diseases with different etiological factors and even side-effects of medications. One paper was found on pre-lingual deafness.

In this paper we report 24 new cases of acquired hearing impairment ranging from mild hearing impairment to severe deafness. The patients were seen in the outpatients clinic over a ten-year period; 16 were female $(67 \%)$ and $8(33 \%)$ were male. The mean age \pm SD was 79 years \pm 11.07 (range: $51-95$ years). Of these 24 patients, 14 had no other mental or physical health problems apart from hearing impairment but the rest had other conditions. Table 1 shows the demographic characteristics of all patients in this study.
Williams, D. R. and Lees, A. J. (2005). Visual hallucinations in the diagnosis of idiopathic Parkinson's disease: a retrospective autopsy study. Lancet Neurology, 4, 605-610. doi:10.1016/S1474-4422(05)70146-0.

\author{
JOANNA M. JefFERIs, ${ }^{1}$ URS P. MOSIMANn, ${ }^{2}$ \\ John-Paul TAYlor ${ }^{3}$ and Michael P. \\ ClaRKE $^{4}$ \\ ${ }^{1}$ Research Fellow, Institute of Neurosciences and \\ Institute for Ageing and Health, Newcastle University, \\ Newcastle Upon Tyne, UK \\ ${ }^{2}$ Professor of Old Age Psychiatry, University of Bern, \\ Switzerland \\ ${ }^{3}$ Wellcome Intermediate Clinical Fellow, Institute for \\ Ageing and Health, Newcastle University, Newcastle \\ Upon Tyne, UK \\ ${ }^{4}$ Consultant Ophthalmologist, Department of \\ Ophthalmology, Royal Victoria Infirmary, Newcastle \\ upon Tyne UK \\ Email: j.m.jefferis@ncl.ac.uk
}

In three cases the onset of musical hallucinations coincided with an alteration in hearing ability due to: (i) proximity to an exploding grenade; (ii) removal of wax from the ear; and (iii) a cochlear implant. In the first case, musical hallucination started in early adult life following exposure to a grenade explosion; in the second case the music was very distinct and familiar to the patient; in the third case having a cochlear implant has helped to cure the musical hallucinations almost completely. Two patients had both hearing and visual impairment resulting in a major decrease in sensory perception. Three of the patients showed signs of mild cognitive impairment and one had had a stroke. Three suffered from depression and one had shingles. The rest of the patients had no significant mental or physical health problems.

One woman reported that the songs in her head held no special significance for her and she did not like them. She had tried playing a CD of her preferred music in a bid to replace something unpleasant with something more to her taste. Unfortunately, when the new tunes appeared in her hallucinations, they were often sung in a distorted and distressing manner.

The musical hallucinations reported by the patients were generally Christmas carols, religious songs, men's choral music, tunes or operatic nonvocal pieces and orchestral music. The musical hallucinations were identified as emanating from the nearby environment and one woman even described it as "those girls singing very loudly upstairs". 
Table 1. Demographic characteristics

\begin{tabular}{lc}
\hline Living alone & $4(17 \%)$ \\
Hearing impairment & $17(71 \%)$ \\
$\quad$ Severe & $6(24 \%)$ \\
$\quad$ Moderate & $1(4 \%)$ \\
$\quad$ Mild & \\
Musical hallucinations & $23(96 \%)$ \\
$\quad$ Bilateral & $1(4 \%)$ \\
$\quad$ Unilateral & \\
Insight into abnormality & $24(100 \%)$ \\
$\quad$ Yes & $24(100 \%)$ \\
Perceived distance & $2(8 \%)$ \\
$\quad$ Nearby & $7(29 \%)$ \\
Hearing + visual impairment & $3(13 \%)$ \\
Presence of psychiatric illness & $1(4 \%)$ \\
Mild cognitive impairment & $3(13 \%)$ \\
Stroke & $0(0 \%)$ \\
Depression & $3(13 \%)$ \\
Tinnitus & \\
Presence of physical illness & \\
\hline
\end{tabular}

In most cases, despite wearing hearing aids and thus with improved hearing, they have continued to hear the musical hallucinations. An interesting question remains whether people who are born deaf can hear musical hallucinations.

We surveyed specialist psychiatrists for the deaf in the UK in 2008 to elicit their experiences of patients with musical hallucinations. About $60 \%$ of the specialists responded to our survey and four cases were reported. Two patients, who have recently received cochlear implants, reported florid musical hallucinations which started prior to the "switch on" of their new implants. For one of the patients this was their only symptom but the other patient also developed other psychotic symptoms. Another two patients, who were pre-lingually deafened, also described musical hallucinations. Both bore the diagnosis of schizophrenia. The first made references to "music in the sky" while the second referred to hearing "drumming". In a phenomenologically oriented paper by Du Feu and McKenna (1999), it was found that approximately half of pre-lingual profoundly deaf patients had auditory hallucinations and in one or two cases these were nonverbal.

Different theories have been proposed to explain the mechanisms of musical hallucinations. The physical origin appears to lie somewhere between the cochlear and the brain itself or in both. Gordon (1997) concluded that ear disorder is a sufficient cause and postulated that the otological basis for the condition is a hyperactive state of the ear.
Lack of stimulation to certain areas of the brain appears to be an important mediator in musical hallucinations, indicated by the association of deafness and musical hallucinations that is already well established (Berrios, 1990). The perceptual "release" theory maintains that the ear must maintain a minimal amount of stimulation in order to suppress spontaneous perceptions. Areas of the cerebral cortex that are redundant because of deafness or isolation are prone to generate abnormal experiences. When the perceptual stimulus falls below the threshold amount, musical hallucinations may develop.

The "release" phenomenon can explain musical hallucination in pre-lingual deafness to some extent, and there must also be another central mechanism that activates the brain areas associated with hearing and music perception. Griffiths (2000) has suggested the involvement of central mechanisms based on amplified musical imagery and activation of cortical networks usually involved in perception and imagery.

Owing to the lack of any database of the demographic characteristics of deaf psychiatric patients over the years, it is not possible to look at the etiological basis of the condition. In addition, due to the very small number of specialist services for deaf patients, and because of the inherent communication issues, it is difficult to estimate how many patients in the UK may have such symptoms but have not been identified by relevant services and so remain undiagnosed/untreated.

Furthermore, some deaf patients do possess some residual hearing, so it is sometimes difficult to determine what is true hearing of music and what is a hallucination. Even profoundly deaf patients with no hearing still have some concept of music owing to their ability to feel vibrations, so again this can complicate the picture.

We hope that the advances in neuroimaging techniques and future research in this fascinating area can provide an answer to this phenomenon. We believe it is worth asking patients with a hearing impairment whether they have had musical hallucinations.

\section{References}

Berrios, G. E. (1990). Musical hallucinations: a historical and clinical study. British Fournal of Psychiatry, 156, 188-194.

Du Feu, M. and McKenna, P. J. (1999). Prelingually profoundly deaf schizophrenic patients who hear voices: a phenomenological analysis. Acta Psychiatrica Scandinavica, 99, 453-459. 
Gordon, A. G. (1997). Do musical hallucinations always arise from the inner ear? Medical Hypotheses, 49, 111-122.

Griffiths, T. D. (2000). Musical hallucinosis in acquired deafness. Phenomenology and brain substrate. Brain, 123, 2065-2076.

Pasquini, F. and Cole, M. G. (1997). Idiopathic musical hallucinations in the elderly, fournal of Geriatric Psychiatry and Neurology, 10, 11-14.
V. M. AZIZ ${ }^{1}$ AND M. ASAAD ${ }^{2}$

${ }^{1}$ Consultant Psychiatrist, St Tydfil's Hospital, Merthyr Tydfil, Wales, UK

${ }^{2}$ Medical student, Oxford University Medical School, Oxford, UK

Email: victoraziz@doctors.org.uk 